\title{
Couple-Based HIV Prevention for Low-Income Drug Users From New York City: A Randomized Controlled Trial to Reduce Dual Risks
}

\author{
Nabila El-Bassel, DSW, ${ }^{*}$ Louisa Gilbert, PhD, * Elwin Wu, PhD, * Susan S. Witte, PhD, * \\ Mingway Chang, PhD, * Jennifer Hill, PhD, $\dagger$ and Robert H. Remien, PhD $\neq$
}

\begin{abstract}
Objective: Dual threats of injection drug use and risky sexual practices continue to increase transmission of HIV and other sexually transmitted Infections (STIs) among drug-using couples in low-income communities in the United States. Two hypotheses were tested: (1) "intervention effect" - whether the HIV risk-reduction intervention provided to the couple or individual partners would be more efficacious in decreasing number of unprotected sexual acts and having a lower cumulative incidence of biologically confirmed STIs over the 12-month follow-up period compared with the attention control condition; and (2) "modality effect" - whether the HIV risk-reduction intervention would be more likely to decrease the number of unprotected sexual acts and have a lower cumulative STI incidence when delivered to a couple compared with the same intervention delivered to an individual.
\end{abstract}

Design: Using a randomized controlled trial, 282 HIV-negative drug-using couples (564 individuals) were randomly assigned to receive either of the following: (1) couple-based risk reduction; (2) individual-based HIV risk reduction, or (3) couple-based wellness promotion, which served as an attention control condition.

Results: Over 12-month follow-up, there was a 30\% reduction in the incidence rate of unprotected acts of intercourse with the study partners compared with participants in the attention control arm. Moreover, over 12-month follow-up there was a $29 \%$ reduction in the same outcome in the couple arm compared with the individual arm with a $41 \%$ reduction at the 12 -month follow-up.

Conclusion: A couple-based approach that addresses drug and sexual risks and targets low-income active drug users may help curb the HIV epidemic.

Key Words: couple-based intervention, drug-using couples, injection drug use, risky sexual practice, sexually transmitted infections

(J Acquir Immune Defic Syndr 2011;58:198-206)

Received for publication February 24, 2011; accepted June 3, 2011

From the *Columbia University School of Social Work, Social Intervention Group, New York, NY; †Columbia University School of International and Public Affairs, New York, NY; and \$Columbia University Department of Clinical Psychiatry, HIV Center for Clinical and Behavioral Studies, New York, NY.

Supported by funding by the National Institutes of Drug Abuse to Dr N.E.B (NIDA R01DA16993).

The authors have no conflicts of interest to disclose.

Correspondence to: Dr Nabila El-Bassel, DSW, Columbia University School of Social Work, 1255 Amsterdam Avenue, New York, NY 10023 (e-mail: ne5@columbia.edu).

Copyright (c) 2011 by Lippincott Williams \& Wilkins

\section{INTRODUCTION}

Dual threats of injection drug use and risky sexual practices continue to fuel transmission of HIV and other sexually transmitted infections (STIs) in low-income urban communities with concentrated HIV epidemics. ${ }^{1}$ Thirty years of research have found that injection and noninjection drug use are associated with having unprotected sex, having concurrent sexual partners, and contracting HIV and other STIs among heterosexuals in committed relationships. ${ }^{2-4}$ Research also suggests that sharing syringes is prevalent among heterosexual injection drug-using couples. ${ }^{5}$ Among heterosexuals, sexual transmission of HIV and other STIs most frequently occurs in the context of intimate relationships. ${ }^{6}$ HIV-negative couples in established relationships report low rates of condom use even when they engage in drug-related or sexual HIV risks with outside partners. ${ }^{7}$ These epidemiological trends underscore the need for HIV prevention approaches that synergistically focus on reducing both drug-related and sexual HIV risk behaviors among HIV-negative drug-involved heterosexual couples.

Research over the past decade suggests the efficacy of a dyadic-focus as the unit of change in reducing sexual HIV risk behaviors among heterosexual couples and decreasing unsafe injection behaviors among heterosexual drug-involved couples. ${ }^{8-13}$ This research suggests that couple-based approaches provide an opportunity for both partners to improve their HIV/STI knowledge, disclose and identify sensitive HIV risks, and to develop mutual goals and joint responsibility for protecting each other from HIV/STIs. ${ }^{9,14,15}$ Despite evidence suggesting the potential efficacy of couple-based HIV prevention approaches, only 4 intervention studies with heterosexual couples have been conducted in the United States. ${ }^{9,15-17}$ These couple-based studies have exclusively focused on sexual risk reduction, targeting mainly HIV serodiscordant and HIV-positive concordant couples. To date, none have focused exclusively on drug-using couples or specifically addressed injection-related risks or drug-related triggers for unsafe sex. ${ }^{18}$

We report on a randomized controlled trial (RCT) designed to address these gaps in couple-based HIV prevention research by focusing exclusively on HIV-negative concordant couples where one or both use drugs. Building on prior couple-based HIV research that resulted in an evidencebased HIV prevention model for couples (connect), intervention components were modified to address dyadic drug risk reduction and drug-related unsafe sex. ${ }^{15}$ This culminated in a couple-based HIV risk-reduction intervention (connect 2) 
specifically for drug-involved HIV-negative concordant heterosexual couples. For this RCT, couples were recruited primarily through street outreach and randomly assigned to receive 1 of 3 interventions as follows: (1) a couple-based HIV risk reduction; (2) an individual-based HIV risk reduction that delivered the same content as the couple-based condition but was provided to either the male or female drug-using partner alone; or (3) a couple-based wellness promotion that served as an attention control condition. This RCT tested 2 primary hypotheses as follows: (1) "intervention effect"-whether the HIV risk-reduction intervention provided to the couple or an individual partner would be more efficacious in decreasing the number of unprotected acts of intercourse and having a lower cumulative incidence of biologically confirmed STIs over the 12-month follow-up period compared with the attention control condition; and (2) "modality effect"-whether the HIV risk-reduction intervention would be more likely to decrease the number of unprotected acts of intercourse and have a lower cumulative STI incidence when delivered to a couple compared with the HIV risk-reduction intervention delivered to an individual.

\section{METHODS}

\section{Study Design and Population}

The Institutional Review Board of Columbia University approved this RCT study, which was conducted between November 2005 and September 2010 in New York City. Participants were recruited through street outreach, homeless shelters, soup kitchens, syringe exchange programs, and wordof-mouth. Most qualified couples came through street outreach. Before screening, potential participants were asked if they would like to complete a brief interview to see if they would be eligible to participate in a study where they would learn ways to protect themselves and their partner from getting HIV and other STIs. If they expressed interest, consent was obtained by recruiters followed by a screening interview that covered sociodemographics and eligibility criteria. Individuals who met initial eligibility criteria were asked to invite their main sexual partner to participate. A letter to their partner introducing the study was given to potential participants. Partners interested in participating were screened. If eligible, the recruiter obtained written informed consent from the partners before enrollment and scheduled the couple for baseline data collection. Participants received monetary compensation for completing the baseline assessment and each follow-up assessment.

Couples were eligible to participate if (1) both were 18 or older and at least 1 partner was 18-40; (2) both tested HIV negative using OraQuick and OraSure assays; (3) both identified each other as their main, regular partner, boy/girlfriend, spouse, lover; (4) both reported that they have been together for at least 6 months and couple status was verified by separately asking each partner standard questions about their relationship (eg, when and where did you first meet your partner?); (5) both intended to remain together for at least one year; (6) at least 1 partner reported using illicit drugs in the prior 90 days and was seeking or in drug treatment; (7) at least
1 partner reported having had unprotected intercourse with the other in the prior 90 days. Additionally, at least 1 partner had to report 1 or more of the following HIV risk criteria: (1) sex with other partners in the prior 90 days; (2) injecting drugs in the prior 90 days; or (3) self-reported being diagnosed with an STI in the prior 90 days. Couples were excluded from the study if either partner (1) reported experiencing severe intimate partner violence in the past year by the other partner, as assessed by subscales of the Revised Conflict Tactics Scale ${ }^{19}$; (2) exhibited a severe cognitive or psychiatric impairment assessed during informed consent; (3) did not sufficiently understand English; (4) reported intentions to conceive a baby in the next year; or (5) reported an intent to relocate in the coming year. Although only 1 partner needed to report having had unprotected anal or vaginal sex with their study partner, in $94 \%$ of the enrolled couples, both partners reported having had at least 1 episode.

\section{Randomization}

Couples were randomized to the couple risk reduction, individual risk reduction, or the couple wellness promotion conditions at the time of their arrival at their first session (within 10 days from baseline, allowing sufficient time to receive HIV and STI test results) (Fig. 1). The gender of the index participant (the one who reported drug use) was used as a blocking factor to balance the proportion of couples with drug-involved female and male participants across study arms. If both partners met drug involvement eligibility criteria, one partner was designated randomly as the index participant.

\section{Procedures}

All 3 intervention conditions consisted of 7 structured 2-hour sessions delivered weekly by a single female or male facilitator - matched to the gender of the index participantwho had at least a bachelor's degree and 2 years of HIV prevention experience. Quality assurance (QA) procedures were implemented by digitally recording all intervention sessions. Recordings and session-specific QA checklists were reviewed to monitor fidelity of implementation of all 3 conditions and to provide corrective feedback to facilitators.

The couple-based HIV risk-reduction intervention was guided by social cognitive theory and a relationship-oriented ecological framework. ${ }^{20,21}$ The core components of this intervention focus on both sexual and drug risk reduction (1) encouraging both partners to disclose and identify mutual drug-related and sexual risks; (2) modeling, role-playing and practicing couple communication, negotiation and problem solving skills that both partners may employ together to reduce their drug-related and sexual risks; (3) practicing technical condom use placement skills along with a broader repertoire of pleasurable safer sex activities and syringe disinfection skills; and (4) enhancing the couple's motivation to protect each other and set mutual risk-reduction goals. Facilitators were trained to validate the relationship's strengths of commitment, love, trust, and empower the dyad to enact protective behaviors. A safe environment was created where sensitive or taboo topics (eg, outside sexual partners) related to a couple's risks could be disclosed and addressed.

The individual risk-reduction intervention covered the same content, skills, and sequencing of activities as the couple 


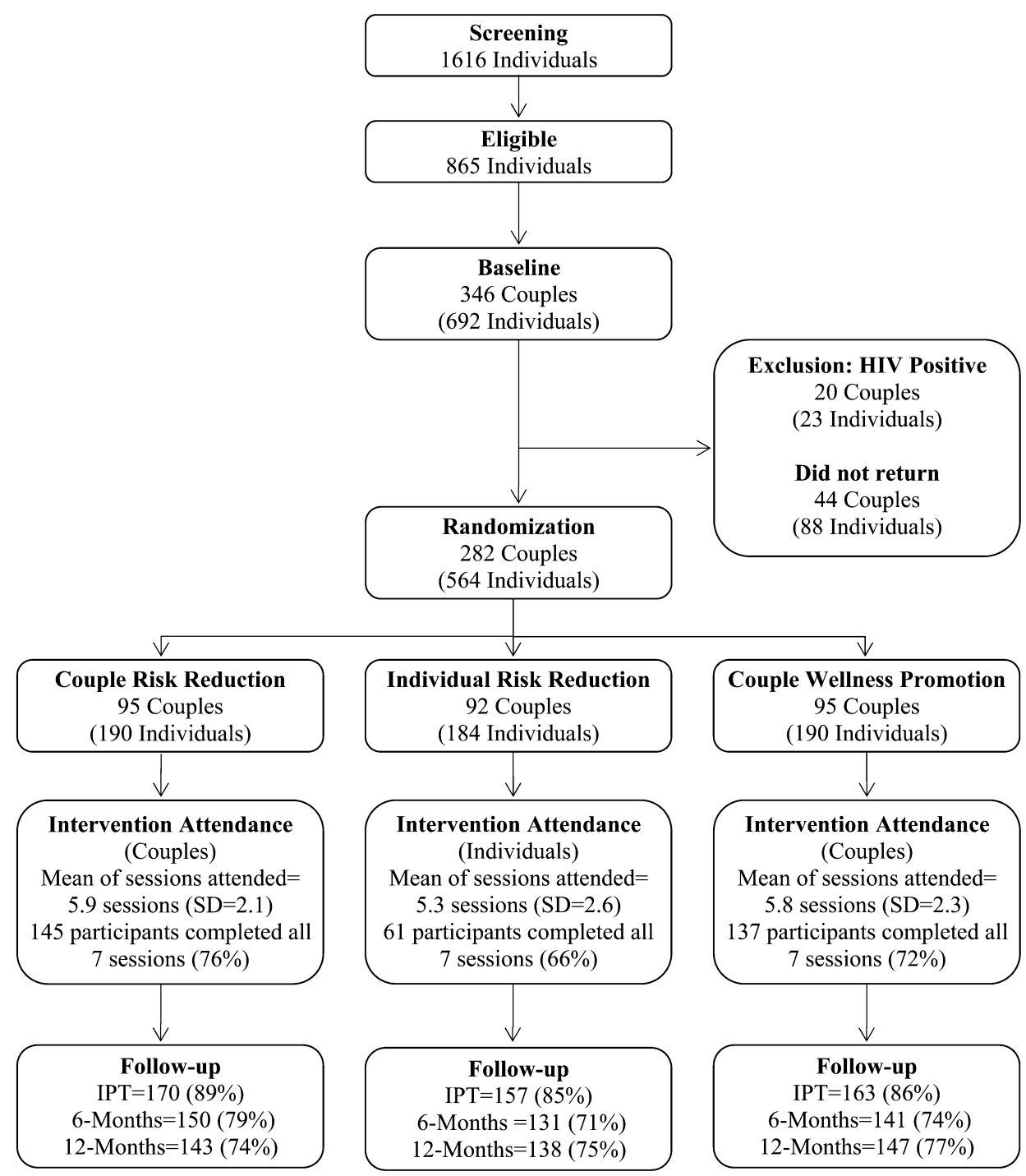

FIGURE 1. CONSORT flow diagram of project connect two. risk reduction but was delivered to either the male or female drug-involved partner in individual sessions by a facilitator of the same gender. In these individual sessions, facilitators were trained to model and role play communication, negotiation, and problem-solving skills with participants and encouraged them to practice these HIV-related skills and share HIV information with their partners in homework activities.

The couple wellness promotion comparison intervention was designed to control for nonspecific effects (eg, Hawthorne effect, couple interaction). Also guided by social cognitive theory, the core components of the intervention consist of 7 weekly 2-hour sessions and employed the same structure as the HIV risk-reduction sessions. The couple wellness promotion comparison intervention focuses on maintaining a healthy diet, promoting physical fitness in daily routines, promoting age-appropriate recommendations for screening for common diseases such as cancers, heart disease, diabetes, etc., improving access to health care services by identifying and addressing service barriers, and learning stress-reduction exercises.
The participants in all 3 conditions provided behavioral and biological outcome data at baseline, immediate postintervention, and at 6-month and 12-month postintervention time points. An audio computer-assisted self-interview (ACASI) was used to collect self-reported data on drugrelated and sexual behavioral outcomes and sociodemographic variables. ACASI provided both audio and video presentation of the questions and response options on a computer enabling participants with low literacy to respond to questions. ACASI was used to collect the participant's self-reported data on sexual behaviors with their study partner and with all other partners in the prior 90 days, including number of vaginal and anal intercourse acts, number of unprotected vaginal or anal intercourse acts with their study partners and with all other partners, consistent condom use (eg, used a condom $100 \%$ of time during vaginal sex), and incidence of concurrent sexual partners in the past 90 days at baseline and all follow-ups. The timeline follow-back method was used to enhance recall of sexual behaviors. The primary behavioral outcome was the number of unprotected acts of vaginal intercourse. The reports 
of conjoint sexual behaviors between couple partners were correlated at a high level of significance $(P<0.001$ for all conjoint sexual behavioral measures). Discrepant reports between 2 partners on conjoint behaviors were handled and treated in the error terms modeling individual's responses based on their couple unit in the multilevel mixed-effect models.

Drug risk behavioral outcomes included whether or not and number of times injection drugs were used in the past 90 days and number or times syringes, cookers, cotton, or rinse water were shared with another user in the past 90 days. In addition, self-reported data on frequency and type of drug use in the past 90 days were collected for the following substances: cocaine, crack, heroin, cannabis, and unprescribed sedatives, opiates, and stimulants.

To complement self-reports of primary behavioral outcomes, we assessed biologically confirmed STI cumulative incidence over the 12-month follow-up period. All participants were asked to provide a self-collected urine specimen after completing the ACASI. Molecular assays of urine specimens were performed for Chlamydia trachomatis and Neisseria gonorrhea using BD ProbeTect. Urine specimens were tested for Trichomonas vaginalis using nucleic acid amplification. ${ }^{22}$ Participants with positive STI test results were referred for single-dose antimicrobial treatment and risk-reduction counseling per Centers for Disease Control and Prevention recommendations. If either partner tested positive for an STI, both were recommended for treatment. If participants tested positive for any STI at any assessment visit, they were considered an incident STI case for that visit. HIV testing was repeated at the 12-month follow-up assessment using OraQuick and OraSure assays.

\section{Statistical Analysis}

Consistent with an intent-to-treat approach, participants were analyzed based on their experimental assignment in estimating intervention effects. To detect a reduction in the primary outcome (number of unprotected vaginal sex acts with a study partner) with effect sizes at least as small as those observed with our prior couple-based and assuming $\alpha=5 \%$ ( 2 sided) and $1-\beta=80 \%$, a sample size of $90-95$ couples per arm was necessary given an anticipated dropout rate of $15 \%$ $20 \%$. To handle nonindependence in observations, multilevel mixed-effects models were employed. Each observation at the first level of the model represents an individual measurement at 1 of the 3 posttreatment time points. Random effect parameters in the model account for dependencies present with each of 2 types of grouping couple and time point. Multilevel mixed-effects Poisson regressions were used to estimate effects of the intervention on the number of unprotected acts of intercourse; corresponding effect estimates are reported as incident rate ratios (IRR) and corresponding 95\% confidence intervals (CIs). Multilevel mixed-effects logistic regressions were used to estimate effects on consistent condom use during vaginal sex, any unprotected anal sex, having concurrent partners, injecting drugs, sharing needles or syringes, and sharing works, cooker, cotton, or rinse water; for these effect estimates, odds ratios and $95 \%$ CIs are reported. All available data on participants who were randomized were used in the models. Due to the low prevalence of STI at baseline and low cumulative incidence over the follow-up period, the biologically assayed outcomes were not included in these multilevel analyses.

Orthogonal contrast coding was used to test separately for intervention effect (couple and individual risk reduction jointly vs. couple wellness promotion) and modality effect (couple risk reduction vs. individual risk reduction) within one model. To test for an intervention effect, the coding for couple risk reduction, individual risk reduction, and health promotion were $+1 / 3,+1 / 3$, and $-2 / 3$, respectively. To test for modality effects, the coding for couple risk reduction, individual risk reduction, and health promotion were $+1 / 2,-1 / 2$ and 0 , respectively. All the multilevel models adjusted for the baseline measure of the outcome and gender.

Our attrition analysis comparing the sociodemographic variables of those who completed all 3 follow-up assessments (completers) versus those who missed 1 or more follow-up assessments (noncompleters) found no differences between completers and noncompleters with respect to age, marital status, income, employment status, whether recently incarcerated or in jail, and whether in substance abuse treatment or use of illicit drugs in the past 30 days. However, completers were more likely than noncompleters to be African American, older, and less likely to be homeless. To assess whether these sociodemographic differences between completers and noncompleters may have influenced the intervention outcomes, we examined intervention outcomes using covariance adjustment for baseline outcome, gender, ethnicity, age, and homelessness. Patterns of significance did not change and point estimates for intervention/modality effects changed less than $1 \%$. Because the point estimates and significance did not change, we presented the results using models that had as few covariates as possible following the principle of parsimony.

This study is registered with ClinicalTrials.gov, number NCT01285349.

\section{RESULTS}

Figure 1 presents a CONSORT flow diagram depicting participant flow: 1616 individuals completed the eligibility screening interview and 865 were eligible; 346 couples (692 individuals) consented and completed baseline interviews and HIV/STI testing. Twenty couples were excluded at baseline because at least 1 partner tested positive for HIV. Of the remaining 326 couples, 282 couples returned for a first session and were randomized ( $87 \%$ participation rate), thus constituting the study sample as follows: 95 couples ( $\mathrm{n}=190$ participants) were randomized to the couple HIV risk-reduction condition; 92 couples $(n=184$ participants) were randomized to the individual HIV risk reduction condition for the index drug-using partner and 95 couples ( $n=190$ participants) were randomized to the couple wellness promotion condition. Attendance at intervention sessions for all 3 conditions was high. Participation rates for completing all 7 sessions of the intervention were as follows: $72 \%$ for couple risk reduction, $66 \%$ for the individual risk reduction, and $76 \%$ for couple wellness promotion. No adverse events were detected by study staff or by QA procedures. We completed 489 immediate postintervention tests (IPTs) follow-up assessments 
averaging an $87 \%$ retention rate, 421 completed their 6 months assessments (75\% retention rate), and 428 individuals completed their 12 months assessments ( $76 \%$ retention rate). Follow-up rates did not vary significantly among the 3 conditions.

Table 1 reports baseline sociodemographic and drug treatment characteristics. The average age of participants was 36.5 years $(\mathrm{SD}=7 \cdot 1)$. The majority identified as black or Latino. Less than half indicated being married. Three-quarters were homeless, and most were unemployed and living below the poverty line. One-fifth $(21 \%)$ reported recent incarceration.

Table 2 presents baseline sexual behaviors and drug risk characteristics. The sample reported engaging in a range of sexual and drug-related risk behaviors, including high incidence of unprotected sex acts with study partners and across all partners. About one-third reported having had sex with other partners during the past 90 days and $16 \%$ reported injecting drugs in the past 30 days. Although only 1 partner needed to report using drugs in the past 30 days to be eligible, $82 \%$ of the participants indicated drug use in the past 30 days. Almost two-thirds (63\%) used crack/cocaine in the past 30 days and $16 \%$ injected drugs in the past 30 days. Baseline characteristics reported in Table 1 did not vary significantly by intervention condition.

Table 3 presents the descriptive statistics of self-reported sexual and drug risk outcomes and biological STI outcomes by time and by condition. Prevalence of the 3 STIs that we tested for was low at the baseline assessment and was even lower at each follow-up assessment. There were only 23 cumulative incident cases of any confirmed new STI over the entire follow-up period. The 12-month follow-up HIV testing only detected seroconversion of 1 individual.

Table 4 presents the results from multilevel analysis examining the effects of intervention (couple and individual risk reduction jointly vs. couple wellness promotion) and the effects of modality (couple risk reduction vs. individual risk reduction) with respect to the sexual risk behavior outcomes after adjusting for the baseline measures of the outcomes and gender. The results indicate that, over the entire follow-up period, there was a $30 \%$ reduction in the incidence rate of unprotected acts of intercourse with their study partners for the participants in the 2 risk-reduction intervention conditions compared with the participants in the couple wellness promotion condition (IRR $=0.70 ; 95 \% \mathrm{CI}=0.53$ to 0.92 ) and a $29 \%$ reduction when comparing couple risk reduction with Individual Risk Reduction (IRR $=0.71 ; 95 \%$ $\mathrm{CI}=0 \cdot 51,0.97)$. These intervention effects were significant at IPT $($ IRR $=0 \cdot 58 ; 95 \% \mathrm{CI}=0 \cdot 38,0 \cdot 88)$ and 6-month follow-up $(\mathrm{IRR}=0.70 ; 95 \% \mathrm{CI}=0.54,0.92)$ but not at 12 -month follow-up (IRR $=0.85 ; 95 \% \mathrm{CI}=0.55,1.32)$. Although the modality effect was not significant at IPT (IRR $=0 \cdot 84 ; 95 \%$ $\mathrm{CI}=0.52,1.35)$, participants in the Couple Risk Reduction condition experienced a $30 \%$ reduction of unprotected acts of intercourse at 6-month follow-up (IRR $=0.70 ; 95 \% \mathrm{CI}=0.51$, $0.96)$ and a $41 \%$ reduction at 12 -month follow-up (IRR $=0.59$; $95 \% \mathrm{CI}=0.35$ to 0.99$)$ compared with individual risk reduction participants.

We also examined effects of the intervention and modality on injecting drug use in the past 90 days among the total sample.

TABLE 1. Sociodemographic Characteristics: All Participants and by Study Arm

\begin{tabular}{|c|c|c|c|c|}
\hline Characteristics & $\begin{array}{c}\text { Total } \\
(\mathrm{n}=563)^{*}\end{array}$ & $\begin{array}{c}\text { Couple Risk } \\
\text { Reduction }(n=190)\end{array}$ & $\begin{array}{c}\text { Individual Risk } \\
\text { Reduction }(\mathrm{n}=\mathbf{1 8 3})\end{array}$ & $\begin{array}{c}\text { Couple Wellness } \\
\text { Promotion }(n=190) \\
\end{array}$ \\
\hline Age (yrs): mean (SD) & $36.5(7.1)$ & $36.1(7.4)$ & $36.3(7.2)$ & $37.0(6.8)$ \\
\hline Year of schooling: mean (SD) & $11.6(2.9)$ & $11.4(3.2)$ & $11.4(2.8)$ & $11.8(2.6)$ \\
\hline \multicolumn{5}{|l|}{ Ethnicity $\dagger$} \\
\hline Black & $275(50 \%)$ & $91(50 \%)$ & $88(49 \%)$ & $96(51 \%)$ \\
\hline Latino & $150(28 \%)$ & $50(28 \%)$ & $52(29 \%)$ & $48(26 \%)$ \\
\hline White & $62(11 \%)$ & $18(10 \%)$ & $23(13 \%)$ & $21(11 \%)$ \\
\hline Other & $61(11 \%)$ & $22(12 \%)$ & $16(9 \%)$ & $23(12 \%)$ \\
\hline \multicolumn{5}{|l|}{ Marital status } \\
\hline Single & $252(45 \%)$ & $90(47 \%)$ & $84(46 \%)$ & $78(41 \%)$ \\
\hline Married & $241(43 \%)$ & $72(38 \%)$ & $78(43 \%)$ & $91(48 \%)$ \\
\hline Divorced/separated/widowed & $70(12 \%)$ & $28(15 \%)$ & $21(11 \%)$ & $21(11 \%)$ \\
\hline \multicolumn{5}{|l|}{ Monthly income } \\
\hline$\leq \$ 400$ & $423(75 \%)$ & $135(71 \%)$ & $139(76 \%)$ & $149(78 \%)$ \\
\hline$\$ 401$ to $\$ 850$ & $99(18 \%)$ & $44(23 \%)$ & $26(14 \%)$ & $29(15 \%)$ \\
\hline$\geq \$ 851$ & $41(7 \%)$ & $11(6 \%)$ & $18(10 \%)$ & $12(6 \%)$ \\
\hline Unemployed & $456(81 \%)$ & $152(80 \%)$ & $152(83 \%)$ & $152(80 \%)$ \\
\hline Homeless & $415(74 \%)$ & $140(74 \%)$ & $138(75 \%)$ & $137(72 \%)$ \\
\hline In jail or incarcerated in the past 90 days $\ddagger$ & $120(21 \%)$ & $30(18 \%)$ & $42(23 \%)$ & $48(25 \%)$ \\
\hline $\begin{array}{l}\text { Attending drug or alcohol } \\
\text { treatment in the past } 90 \text { days } \S\end{array}$ & $267(48 \%)$ & $98(52 \%)$ & $84(46 \%)$ & $85(45 \%)$ \\
\hline \multicolumn{5}{|c|}{$\begin{array}{l}\text { *The data for one man was lost due to computer failure. } \\
\dagger \text { The valid sample sizes were (couple risk reduction, individual risk reduction, couple wellness promotion) }=(181,179,188) \text {. } \\
\text { †The valid sample sizes were (couple risk reduction, individual risk reduction, couple wellness promotion) }=(190,181,190) \text {. } \\
\S \text { The valid sample sizes were (couple risk reduction, individual risk reduction, couple wellness promotion) }=(189,183,190) \text {. }\end{array}$} \\
\hline
\end{tabular}


TABLE 2. Relationship Characteristics and HIV Risks: All Participants and by Study Arm

\begin{tabular}{|c|c|c|c|c|}
\hline Characteristics & $\begin{array}{c}\text { Total } \\
(\mathrm{n}=\mathbf{5 6 3})^{*}\end{array}$ & $\begin{array}{c}\text { Couple Risk } \\
\text { Reduction }(n=190)\end{array}$ & $\begin{array}{c}\text { Individual Risk } \\
\text { Reduction }(\mathrm{n}=\mathbf{1 8 3})\end{array}$ & $\begin{array}{l}\text { Couple Wellness } \\
\text { Promotion }(n=190)\end{array}$ \\
\hline $\begin{array}{l}\text { Length of relationship (yrs) } \\
\text { with study partner }\end{array}$ & $6.4(6.3)$ & $6.2(6.0)$ & $6.3(5.8)$ & $6.6(7.1)$ \\
\hline $\begin{array}{l}\text { Number of unprotected vaginal sex acts with } \\
\text { study partner in the past } 90 \text { days }\end{array}$ & $42.0(49.8)$ & $37.5(38.8)$ & $43.2(49.2)$ & $45.2(59.2)$ \\
\hline $\begin{array}{l}\text { Number of unprotected vaginal sex acts across } \\
\text { all partners in the past } 90 \text { days }\end{array}$ & $44.3(51.8)$ & $40.6(41.7)$ & $44.9(50.1)$ & $47.5(61.6)$ \\
\hline $\begin{array}{l}\text { Number of anal sex acts with study } \\
\text { partner in the past } 90 \text { days }\end{array}$ & $3.6(10.3)$ & $4.0(9.1)$ & $2.8(9.8)$ & $4.0(11.7)$ \\
\hline $\begin{array}{l}\text { Number of unprotected anal sex acts with study } \\
\text { partner in the past } 90 \text { days }\end{array}$ & $3.3(10.0)$ & $3.4(8.8)$ & $2.4(9.3)$ & $3.9(11.7)$ \\
\hline Number of sexual partners in the past 90 days & $2.2(5.0)$ & $2.6(3.6)$ & $2.5(7.7)$ & $1.6(1.8)$ \\
\hline Illicit drug use in the past 30 days & $459(82 \%)$ & $162(85 \%)$ & $143(78 \%)$ & $154(81 \%)$ \\
\hline Binge drinking in the past 30 days & $212(38 \%)$ & $83(44 \%)$ & $68(37 \%)$ & $61(32 \%)$ \\
\hline Injected drugs in the past 30 days & $91(16 \%)$ & $31(16 \%)$ & $33(18 \%)$ & $27(14 \%)$ \\
\hline Shared needle or syringes in the past 30 days & $35(6 \%)$ & $12(6 \%)$ & $13(7 \%)$ & $10(5 \%)$ \\
\hline $\begin{array}{l}\text { Shared works, cooker, cotton, or rinse } \\
\text { water in the past } 30 \text { days }\end{array}$ & $37(7 \%)$ & $12(6 \%)$ & $14(8 \%)$ & $11(6 \%)$ \\
\hline Any STI & $24(4 \%)$ & $6(3 \%)$ & $11(6 \%)$ & $7(4 \%)$ \\
\hline
\end{tabular}

*The data for one participant was lost due to computer failure.

$\dagger P<0.05$ ( $\chi^{2}$ test or $t$ test).

$\ddagger$ All the participants had HIV/STI test at the Baseline. The valid sample sizes were (Couple Risk Reduction, Individual Risk Reduction, Couple Wellness Promotion) $=(190,184,190)$.

Although the effects were not significant for intervention or modality at the 0.05 level, most likely due to the relatively low prevalence of injection drug use $(16 \%, \mathrm{n}=91)$, a promising effect for injection drug use over the follow-up period was found at the $0 \cdot 1$ level when comparing the 2 risk-reduction interventions to the wellness promotion condition.

\section{CONCLUSIONS}

To our knowledge, this is the first RCT to test the efficacy of a couple-based HIV prevention intervention for drug-using HIV negative couples. Street outreach recruitment methods that targeted drug using spots in low income urban neighborhoods, homeless shelters and soup kitchens, resulted in an unique sample, the majority of whom were homeless and active crack cocaine users, engaging in multiple sexual and drug risk behaviors. The high participation, attendance, and retention rates achieved in this trial demonstrate the feasibility of engaging impoverished street-based drug users, who remain at very high risk of HIV/STIs in a couple-based behavioral intervention. Furthermore, the observed magnitude and consistency of findings for the primary behavioral outcome and across the risk-reduction measures support the 2 major hypotheses and provide robust evidence of efficacy of the couple-based HIV prevention intervention.

Statistically significant effect sizes averaged over the 1-year follow-up period were observed for the primary behavioral outcome for both study hypotheses. When both risk-reduction intervention arms were combined, there was a 30\% reduction in the incidence rate of unprotected acts of intercourse with the study partners compared with participants in the couple wellness promotion arm over the follow-up period. Although the effect of the intervention was significant for the overall follow-up period and at IPT and 6 months follow-up points, it lost significance at 12 months. With regards to modality effects, participants assigned to couple risk reduction had a $29 \%$ reduction in incidence of unprotected sex with study partner over the 12-month follow-up period compared with individual risk-reduction participants. In contrast to the typical attenuating effects over time observed for most behavioral interventions in trials, the significance and effect sizes strengthened over time. At IPT, there was no significant difference in incidence of unprotected sex acts between the 2 HIV intervention conditions, however, by the 12 -months follow-up, there was a $41 \%$ reduction compared with participants assigned to the individual condition. The strengthening of effects over time was also found for the 
TABLE 3. Summary of Sexual Behavior, Injection Drug Use, and Biological Outcomes at Baseline, IPT, and 6-Month and 12-Month Follow-up Assessments

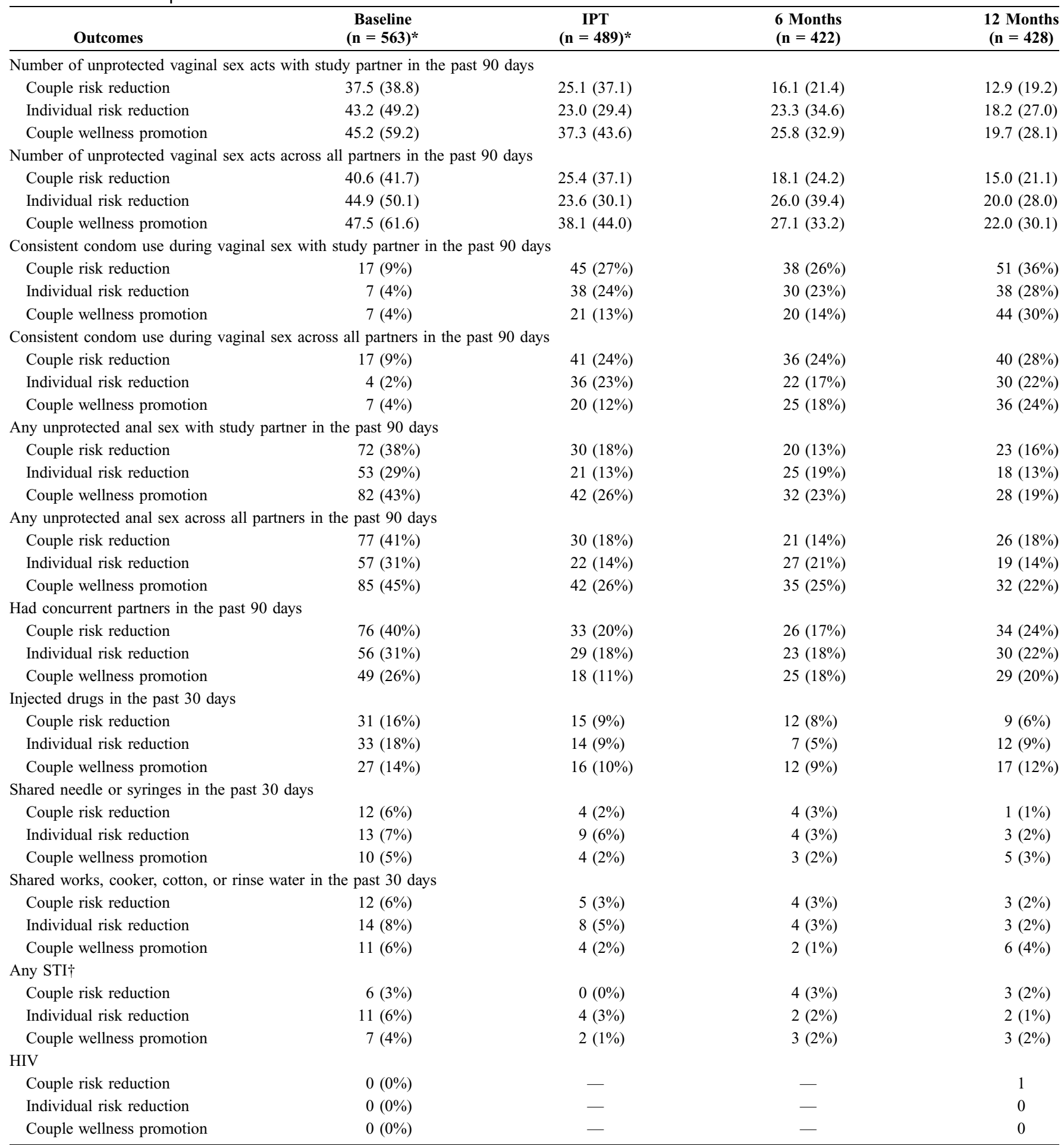

*The data for one participant was lost due to computer failure.

$\dagger$ The sample size was 564 for the baseline and 490 for the IPT. 


\begin{tabular}{|c|c|c|c|c|}
\hline & Entire Follow-up & IPT & 6 Months & 12 Months \\
\hline \multicolumn{5}{|c|}{ Number of unprotected vaginal sex acts with study partner (IRR) } \\
\hline Intervention (risk reduction vs. wellness promotion) & $0.70(0.53 \text { to } 0.92)^{*}$ & $0.58(0.38 \text { to } 0.88)^{*}$ & $0.70(0.54$ to 0.92$) \dagger$ & $0.85(0.55$ to 1.32$)$ \\
\hline Modality (couple risk vs. individual risk) & $0.71(0.51$ to 0.97$) \dagger$ & $0.84(0.52$ to 1.35$)$ & $0.70(0.51$ to 0.96$) \dagger$ & $0.59(0.35$ to 0.99$) \dagger$ \\
\hline \multicolumn{5}{|c|}{ Number of unprotected vaginal sex acts across all partners (IRR) } \\
\hline Intervention (risk reduction vs. wellness promotion) & $0.69(0.54 \text { to } 0.88)^{*}$ & $0.59(0.41 \text { to } 0.86)^{*}$ & $0.69(0.54 \text { to } 0.88)^{*}$ & $0.81(0.55$ to 1.19$)$ \\
\hline Modality (couple risk vs. individual risk) & $0.74(0.56$ to 0.99$) \dagger$ & $0.89(0.57$ to 1.37$)$ & $0.74(0.56$ to 0.98$) \dagger$ & $0.62(0.39$ to 0.98$) \dagger$ \\
\hline \multicolumn{5}{|c|}{ Consistent condom use during vaginal sex with study partner (OR) } \\
\hline Intervention (risk reduction vs. wellness promotion) & $2.07(1.25 \text { to } 3.45)^{*}$ & $4.09(1.76 \text { to } 9.49)^{*}$ & $2.16(1.29 \text { to } 3.61)^{*}$ & $1.14(0.54$ to 2.40$)$ \\
\hline Modality (couple risk vs. individual risk) & $1.18(0.68$ to 2.03$)$ & $0.94(0.40$ to 2.17$)$ & $1.18(0.68$ to 2.02$)$ & $1.48(0.63$ to 3.48$)$ \\
\hline \multicolumn{5}{|c|}{ Consistent condom use during vaginal sex across all partners (OR) } \\
\hline Intervention (risk reduction vs. wellness promotion) & $1.56(0.98$ to 2.47$)$ & $2.85(1.34 \text { to } 6.06)^{*}$ & $1.61(1.01$ to 2.55$) \dagger$ & $0.91(0.45$ to 1.81$)$ \\
\hline Modality (couple risk vs. individual risk) & $1.19(0.71$ to 1.98$)$ & $0.96(0.44$ to 2.07$)$ & $1.20(0.72$ to 1.99$)$ & $1.50(0.66$ to 3.38$)$ \\
\hline \multicolumn{5}{|l|}{ Any unprotected anal sex with study partner (OR) } \\
\hline Intervention (risk reduction vs. wellness promotion) & $0.65 \dagger(0.42$ to 1.02$) \ddagger$ & $0.52(0.27$ to 1.03$) \ddagger$ & $0.66(0.42$ to 1.03$) \ddagger$ & $0.83(0.40$ to 1.72$)$ \\
\hline Modality (couple risk vs. individual risk) & $0.90(0.52$ to 1.56$)$ & $0.85(0.37$ to 1.96$)$ & $0.90(0.52$ to 1.56$)$ & $0.95(0.39$ to 2.30$)$ \\
\hline \multicolumn{5}{|l|}{ Any unprotected anal sex across all partners (OR) } \\
\hline Intervention (risk reduction vs. wellness promotion) & $0.61(0.39$ to 0.94$) \dagger$ & $0.53(0.27$ to 1.04$) \neq$ & $0.61(0.40$ to 0.95$) \dagger$ & $0.70(0.35$ to 1.42$)$ \\
\hline Modality (couple risk vs. individual risk) & $0.83(0.48$ to 1.42$)$ & $0.73(0.32$ to 1.66$)$ & $0.83(0.49$ to 1.43$)$ & $0.95(0.40$ to 2.25$)$ \\
\hline \multicolumn{5}{|l|}{ Had concurrent partners $(\mathrm{OR})$} \\
\hline Intervention (risk reduction vs. wellness promotion) & $1.06(0.71$ to 1.58$)$ & $1.36(0.71$ to 2.61$)$ & $1.08(0.72$ to 1.61$)$ & $0.85(0.46$ to 1.56$)$ \\
\hline Modality (couple risk vs. individual risk) & $0.82(0.52$ to 1.29$)$ & $0.84(0.42$ to 1.68$)$ & $0.82(0.52$ to 1.28$)$ & $0.80(0.40$ to 1.62$)$ \\
\hline
\end{tabular}

couple-based condition with regard to the incidence of unprotected sex across all main and all other sexual partners. Furthermore, statistical models with additional covariate adjustments of sociodemographic characteristics also yielded the same significant findings.

These findings suggest that when couples receive the intervention together, they are more likely to improve and sustain positive protective behaviors over time compared with when 1 partner receives the intervention alone. Further research is needed to explain what mediators or mechanisms explain the robust maintenance of intervention effects for the couple modality compared with the individual modality. The strong maintenance of intervention effects over time suggests superior cost-effectiveness of delivering this relationshipbased intervention using a couple-based modality over the individual modality.

Although the intervention effects on injection drug risk behavior was not significant at the 0.05 level, promising effects were found at the $0 \cdot 1$ level, underscoring the potential utility of employing a couple-based approach in reducing drug risks. The study was not powered to examine the effects of the intervention on incidence of HIV and STIs. Among the 428 12-month follow-up participants, 1 participant assigned to the couple risk reduction seroconverted, which translates to 234 positives per 100,000 population. (This rate was much greater than the estimated new HIV incidence rate of $23 \cdot 2$ per 100,000 population for the US general population in $2006 .{ }^{23}$ Incidence of STIs was low at 23 (4\%) over the 12-month period, and these rates were similar across conditions. The lack of significant biologically STI outcome is a limitation of this study. How individuals who engage in HIV risks remain HIV seronegative is a conundrum that has not been adequately studied. It requires future studies with larger sample sizes to be suitably powered for a sufficient number of HIV and STI transmissions to elucidate this mechanism of great public health and biomedical importance.

The study has a number of strengths. It employed an RCT, with a dose and modality equivalent control group and an active comparison relationship-based intervention that covered the same content as the experimental condition but was delivered to 1 partner. The street outreach recruitment methods resulted in a unique sample of HIV-negative drug users, most of whom were homeless, engaging in multiple risky behaviors, and not in drug treatment despite reporting heavy use of crack cocaine and other drugs. The large number of homeless people and use of multiple settings may limit its generalizeability to any one setting. However, the use of street outreach recruitment methods may be generalizable to a number of settings that serve similar vulnerable populations who remain at high risk of HIV, including syringe exchange programs, street outreach programs, homeless shelters, and criminal justice settings. Selection bias arising from refusals to participate and attrition may have influenced study results. Nonetheless, the relatively high participation, retention, and attendance rates achieved in this study despite the fact that the majority of participants were homeless demonstrate the feasibility and importance of including participants without 
permanent addresses, who have been typically excluded in most trials with drug users.

In sum, the findings draw attention to an effective intervention strategy that can be scaled-up for drug-involved couples in harm-reduction programs, drug treatment, and criminal justice systems. HIV prevention with at-risk heterosexual drug users in low-income urban neighborhoods with concentrated epidemics remains a priority and essential to achieving the $25 \%$ reduction of new infections and reducing HIV/AIDS-related racial/ethnic disparities 2015 goals of our recent national HIV strategic plan. A couple-based approach to primary prevention of HIV that addresses both drug and sexual risks and targets low-income urban active drug users may help curb the HIV epidemic in the United States and may have dissemination potential to address the global HIV epidemic.

\section{REFERENCES}

1. Centers for Disease Control and Prevention (CDC). Subpopulation estimates from the HIV incidence surveillance system: United States, 2006. MMWR Morb Mortal Wkly Rep. 2008;57:985-989.

2. Bowen A, Williams M, Dearing E, et al. Male heterosexual crack smokers with multiple sex partners: between-and within-person predictors of condom use intention. Health Educ Res. 2006;21:549-559.

3. Logan TK, Leukefeld C. HIV risk behavior among bixsexual and heterosexual drug users. J Psychoactive Drugs. 2000;32:239-248.

4. Rasch R, Weisen C, Weschberg W, et al. Patterns of HIV risk and alcohol use among African-American crack abusers. Drug Alcohol Depend. 2000; 58:259-266

5. Wagner KD, Hudson SM, Latka MH, et al. The effect of intimate partner violence on receptive syringe sharing among young female injection drug users: an analysis of mediation effects. AIDS Behav. 2009;13:217-224.

6. Bowen A, Williams M, Dearing E, et al. Male heterosexual crack smokers with multiple sex partners: between-and within-person predictors of condom use intention. Health Educ Res. 2006;21:549-559.

7. Misovich SJ, Fisher JD, Fisher WA. Close relationships and elevated HIV risk behavior: Evidence and possible underlying psychological processes. Rev Gen Psychol. 1997;1:72-107.

8. Burton J, Darbes LA, Operario D. Couples-focused behavioral interventions for prevention of HIV: systematic review of the state of evidence. AIDS Behav. 2010;14:1-10.
9. El-Bassel N, Jemmott JB, Wingood GM, et al. NIMH multisite eban HIV/ STD prevention intervention for African American HIV serodiscordant couples: a cluster randomized trial. Arch Intern Med. 2010;170: 1594-1601.

10. El-Bassel N, Witte S, Gilbert L, et al. Long-term Effects of an HIV/STI Sexual Risk Reduction Intervention for Heterosexual Couples. Aids Behav. 2005;9:1-13.

11. El-Bassel N, Terlikbayeva A, Pinkham S. HIV and Women who use drugs: double risk, double neglect. A call to multi-level gender-focused HIV prevention strategies. Lancet. 2010;376:312-313.

12. Coates T, Richter L, Caceres C. Behavioural strategies to reduce HIV transmission: how to make them work better. Lancet. 2008;372:669-684.

13. Gilbert L, El-Bassel N, Terlikbayeva A, et al. Couple-based HIV prevention for injecting drug users in Kazakhstan: a pilot intervention study. Int J Interv Community. 2010;38:162-176.

14. El-Bassel N, Witte S, Gilbert L, et al. HIV prevention for intimate couples: a relationship-based model. Fam Syst Health. 2001;19:379-395.

15. El-Bassel N, Witte S, Gilbert L, et al. Long-term effects of an HIV/STI sexual risk reduction intervention for heterosexual couples. Aids Behav. 2005;9:1-13.

16. Harvey SM, Kraft JM, West SG, et al. Effects of a health behavior change model-based HIV/STI prevention intervention on condom use among heterosexual couples: a randomized trial. Health Educ Behav. 2009;36: 878-894.

17. Koniak-Griffin D, Lesser J, Henneman T. HIV prevention for Latino adolescent mothers and their partners. West J Nurs Res. 2008;30:724.

18. Degenhardt L, Mathers B, Vickerman P, et al. HIV in people who use drugs 2: prevention of HIV infection for people who inject drugs: why individual, structural, and combination approaches are needed. Lancet. 2010;376:285-301.

19. Straus MA, Hamby SL, Boney-McCoy S, et al. The Revised Conflict Tactics Scales (CTS2): development and preliminary psychometric data. J Fam Issues. 1996;17:283-316.

20. Bandura A. A social cognitive approach to the exercise of control of AIDS infection. In: DiClemente R, ed. Adolescents and AIDS: A Generation in Jeopardy. Newbury Park, CA: Sage; 1992.

21. Bronfenbrenner U. The ecology of human development. Am Psychol. 1979;32:513-531.

22. Mayta H, Gilman RH, Calderon MM, et al. 18S ribosomal DNA-based PCR for diagnosis of Trichomonas vaginalis. J Clin Microbiol. 2000;38: 2683-2687.

23. Centers for Disease Control and Prevention (CDC). Subpopulation estimates from the HIV incidence surveillance system: United States, 2006. MMWR Morb Mortal Wkly Rep. 2008;57:985-989. 